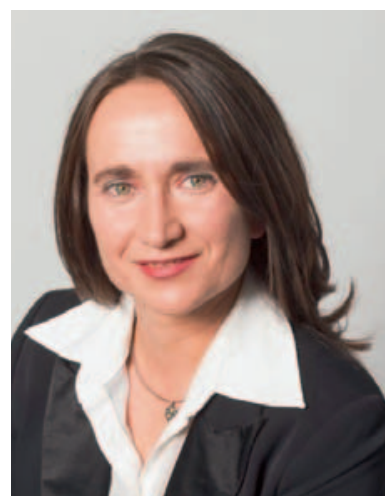

Dr. Evelyn Wolfram,

Wädenswil, Schweiz

\section{Der Medizinnobelpreis 2015 - Auswirkungen auf die Phytopharmazie?}

Während ich dieses Editorial vonseiten der Fachgruppe Phytopharmazie an der Zürcher Hochschule für Angewandte Wissenschaften (ZHAW), Wädenswil, für die vorliegende Ausgabe der SCHWEIZERISCHE ZeITsChrIfT FÜR GANZHeitsmedizin verfasste, liess mich eine Neuigkeit innehalten und beschliessen, das ursprüngliche Thema, das ich mir vorgenommen hatte, zu ändern:

Es war die diesjährige Vergabe des Nobelpreises für Physiologie oder Medizin des Karolinska Institutet in Stockholm am 5. Oktober 2015.

Die Neue Zürcher Zeitung (NZZ) titelte: «Medizinnobelpreis 2015: Drei Parasiten- und Malariaforscher ausgezeichnet» [1]. Die Wochenzeitung Die ZEIT schrieb: «Medizin-Nobelpreis: Schluss mit den Schmarotzern! Einnisten, vermehren, töten: Parasiten leben auf unsere Kosten. Sie lösen Seuchen wie Malaria aus. Der Medizin-Nobelpreis geht an drei Kämpfer gegen Einzeller und Würmer» [2].

Viele andere Schlagzeilen um diese Nachricht setzten die Prioritäten ähnlich - der Nobelpreis war aus medialer Perspektive an Forschende verliehen worden, die der Menschheit Waffen für die Bekämpfung von lebensbedrohlichen, parasitären Erregern von Malaria, Flussblindheit und Elefantensyndrom beschert hatten.

Leider verfehlt dieser erste Blick den Kern der wissenschaftlichen Arbeiten, die mit der höchsten weltweiten Auszeichnung für medizinische Forschung geehrt wurden: Alle drei Forschenden hatten ihre Entdeckungen hoch- wirksamer Medikamente der Präsenz von Naturstoffen zu verdanken, welche die Evolution hervorgebracht hatte. Nach der Würdigung der Entdeckung des Penicillins durch Alexander Fleming (Medizinnobelpreis 1945) und des Streptomycins - ein Antibiotikum aus Streptomyceten durch Selman Abraham Waksman (Medizinnobelpreis 1952) gab es lange keine Auszeichnung mehr im Bereich Wirkstoffe, und nun sind es wieder die Naturstoffe, die offenbar die Jury der höchsten medizinwissenschaftlichen Auszeichnung überzeugten [3].

Im Fall von Satoshi Ômura aus Japan und William C. Campbell aus den USA stammten die Wirkstoffe gegen Würmer aus Bodenmikroben. Die Zusammenarbeit dieser beiden Forscher und die gemeinsame Ehrung der Jury spiegelt die klassische und notwendige Interdisziplinarität der Wirkstoffforschung aus der Natur wider: Der Naturstoffforscher Ōmura vermehrte eine Vielzahl möglicherweise bei der Parasitenbekämpfung vielversprechenden Streptomyceten-Spezies im Labor, die dann von Campbell getestet wurden, um die am stärksten wirksamen Biomasse-Extrakte herauszufiltern. Dadurch wurde die Aufklärung und Herstellung des Moleküls Avermectin möglich, das in den Studien die höchste Wirksamkeit aufwies. Ein Beispiel für viele Wirkstoffe, die nicht nur aus rein chemisch-synthetischen Quellen stammen, sondern die durch die Evolution selbst hervorgebracht wurden und auf ihre Entdeckung durch Naturforschende warten - und davon, so wird geschätzt, gibt es noch sehr viele ... [4].

Die Auszeichnung der Chinesin Youyou Tu für ihre Entdeckung des Malariawirkstoffs Artemisinin (Qinghaosu) aus dem Einjährigen Beifuss (Qinghao - Artemisia annua) ist für die Ganzheitsmedizin von besonderem Interesse. Youyou Tu ist Professorin für Traditionelle Chinesische Medizin (TCM) auf Lebenszeit an der Chinesischen Akademie für Chinesische Medizinwissenschaften (ehemals Chinesische Akademie für Traditionelle Chinesische Medizin) in Peking [5]. Sie ist die erste Nobelpreisträgerin für Physiologie oder Medizin aus China.

Der Artikel in Die ZEIT bringt es mit einem Satz auf den Punkt, der mich als Phytopharmazeutin besonders anspricht: «Hätte die Chinesin Youyou Tu, Professorin für Traditionelle Chinesische Medizin, nicht in den Siebzigerjahren dem «Einjährigen» Beifuss (Anmerkung der Autorin: Die ZEIT schrieb irrtümlich «Einblättriger» Beifuss) sein Geheimnis - den Wirkstoff Artemisinin - entlockt, wären Millionen mehr Menschen gestorben. An den Folgen von vielleicht nur einem Mückenstich» [2]. Dahinter steckt aber eine eher langwierige Vorgeschichte der west-

\section{KARGER}

Fax +497614520714

Information@Karger.com

www.karger.com (c) 2015 S. Karger GmbH, Freiburg

1015-0684/15/0276-0320\$39.50/0
Dr. Evelyn Wolfram

Fachgruppe Phytopharmazie

Institut für Biotechnologie, ZHAW Wädenswil

Grüental, Postfach, 8820 Wädenswil, Schweiz

evelyn.wolfram@zhaw.ch 
lichen Ignoranz gegenüber der bahnbrechenden Therapie aus der fernöstlichen traditionellen Medizin, wie ich schon nach kurzer Recherche feststellen konnte. Der Ursprung der Entdeckung in der Zeit von 1969 bis 1972 lag im Studium von über 600 TCM-Rezepturen im Rahmen eines nationalen chinesischen Projekts zur Entwicklung von hochwirksamen Malariamedikamenten ohne Resistenzen in den Jahren der Kulturrevolution und des Vietnamkriegs $[6,7]$. Darunter fand $\mathrm{Tu}$, gemäss einer GEO-Reportage aus dem Jahr 2005, den Hinweis auf Qinghao, das 168 v. Chr. erstmals erwähnt wurde [6]. 1972 lag dann der Wirkstoff Artemisinin vor, der später, wie oft in der Naturstoffchemie, noch chemisch optimiert wurde. Jedoch wurde erst 2001 ein Artemisinin-Derivat von der Weltgesundheitsorganisation in einer Kombinationstherapie offiziell empfohlen und dann erst 2006 in die erste Ausgabe der MalariaBehandlungsrichtlinien aufgenommen (gemäss [8]). Leider ist auch dieser Wirkstoff nicht ohne Resistenzen geblieben, da die Evolution eben jeder Waffe eine neue Verteidigungsstrategie entgegenzusetzen versucht. Möglicherweise ist dabei auch die Isolierung des Wirkstoffs, wie es in der klassischen pharmazeutischen Wirkstoffentwicklung üblich ist, das problematische Element. Möglicherweise könnte die Gabe im Multikomponentensystem eines Teeaufgusses oder Extrakts die bessere Variante sein. Hierzu gibt es in den Literaturdatenbanken bereits Hinweise, dass Untersuchungen zu diesen Überlegungen derzeit angestellt werden $[9,10]$. Die Behandlung dieser Thematik und eine fundierte Darstellung des Stands der verfügbaren Literatur würden aber den Rahmen dieses Editorials sprengen.

Sie und mich, liebe Leserinnen und Leser, die der ganzheitlichen Medizin und der Evaluierung und Anwendung traditionellen medizinischen Wissens mehr zugewandt sind als der Erforschung von Einzelsubstanzen, soll nach- folgendes Zitat von Youyou Tu bestärken, weiterhin die traditionellen medizinischen Schätze zu heben, denn hier steckt ein grosses Potenzial für die Medizin. Es stammt aus dem in Nature Medicine erschienenen Artikel von Tu zu ihrer Geschichte der Entdeckung von Qinghaosu (Artemisinin) [5]:

\footnotetext{
«The examples cited here represent only a sliver of the gifts or potential gifts Chinese medicine has to offer. It is my dream that Chinese medicine will help us conquer life-threatening diseases worldwide, and that people across the globe will enjoy its benefits for health promotion.»
}

Diese Aussage bezogen auf das chinesische Medizinsystem ist aus meiner Sicht durchaus auch auf die Schätze der weltweit vorhandenen menschlichen Heiltraditionen, welche die Nutzung von Naturstoffen beinhalten, übertragbar.

Für mich persönlich ist der diesjährige Medizinnobelpreis für die drei Naturstoffforscher eine grosse Motivation, in meinem Fachgebiet Phytopharmazie selbstbewusst und «parallel-komplementär» zur eher schulmedizinisch betriebenen pharmazeutischen Forschung weiterzuarbeiten. Mit einem Nobelpreis auf dem Gebiet der Naturstoffforschung im Rücken erhält der Zugang zu Forschungsmitteln und Projektförderungen in diesem Bereich hoffentlich Aufwind.

Für die westliche, europäische Phytotherapie wünsche ich mir, dass sie ihren komplementären Platz immer unverkrampfter in der medizinischen Therapiepalette einnehmen kann, so wie es in der TCM in China und KampoMedizin in Japan schon lange praktiziert wird. Vielleicht kann der Nobelpreis von Youyou Tu auch hier etwas bewegen. Herzliche Gratulation und herzlichen Dank vonseiten der SchweIZerische ZeItschrift FÜr GanZHEITSMEDIZIN und von mir persönlich für diese wertvolle Vorreiterrolle, die uns auch in Zukunft begleiten und motivieren wird.

\section{Literatur}

1 www.nzz.ch/wissenschaft/medizin/nobelpreismedizin-2015-1.18622863 (zuletzt aufgerufen am 07.10.15).

2 www.zeit.de/thema/nobelpreise-2015 (zuletzt aufgerufen am 07.10.15).

3 Wikipedia: Liste der Nobelpreisträger für Physiologie oder Medizin. https://de.wikipedia. org/wiki/Liste_der_Nobelpreistr\%C3\%A4ger f\%C3\%BCr_Physiologie_oder_Medizin (zuletzt aufgerufen am 07.10.2015).

4 Luo Y, Cobb RE, Zhao H: Recent advances in natural product discovery. Curr Opin Biotechnol 2014;30:230-237.
$5 \mathrm{Tu}$ Y: The discovery of artemisinin (qinghaosu) and gifts from Chinese medicine. Nat Med 2011;17:1217-1220. www.nature.com/nm/ journal/v17/n10/full/nm.2471.html.

6 Bökemeier R: Ein Kraut wirkt Wunder. GEO Magazin 06/06. www.geo.de/GEO/natur/einkraut-wirkt-wunder-50716.html?t=print (zuletzt aufgerufen am 07.10.15).

7 Hsu E: Reflections on the 'discovery' of the antimalarial qinghao. Br J Clin Pharmacol 2006; 61:666-670.

8 Weltgesundheitsorganisation (WHO): Guidelines for the treatment of malaria, ed 3. 2015. http://apps.who.int/iris/bitstream/10665/ 162441/1/9789241549127_eng.pdf?ua=1 (zuletzt aufgerufen am 07.10.15).
9 Mouton J, Jansen O, Frédérich M, van der Kooy F: Is artemisinin the only antiplasmodial compound in the Artemisia annua tea infusion? An in vitro study. Planta Med 2013;79: 468-470.

10 Suberu JO, Gorka AP, Jacobs L, Roepe PD, Sullivan N, Barker GC, Lapkin AA: Anti-plasmodial polyvalent interactions in Artemisia annua $\mathrm{L}$. aqueous extract - possible synergistic and resistance mechanisms. PLoS One 2013; 8:e80790. 